\title{
Swiss Medical Board oder: «von der Angemessenheit des PSA-Tests»
}

Swiss Medical Board

\section{Unerwähnt lässt die Task Force, dass zur Beurteilung auch drei externe schweizerische Fachspezialisten beigezogen wurden.}

Das Swiss Medical Board hat am 31. Oktober 2011 einen Fachbericht zur Rolle des PSA-Testes bei der Früherkennung des Prostatakarzinoms veröffentlicht. Damit will es zur Klärung der Angemessenheit dieses umstrittenen Tests und zu einem optimalen Einsatz der verfügbaren Mittel beitragen [1]. In der Folge hat die «Task Force Prostatakarzinom der Schweizerischen Gesellschaft für Urologie» in der Ausgabe 51/52/2011 der Schweizerischen Ärztezeitung eine pointierte Stellungnahme mit dem Titel «Swiss Medical Board oder: das Kind mit dem Bade ausschütten» veröffentlicht, worin dem Medical Board «gravierendste Unzulänglichkeiten bei der PSA-Empfehlung» vorgeworfen werden [2].

Korrespondenz: Sekretariat Trägerschaft Swiss Medical Board Obstgartenstrasse 19/21 CH-8090 Zürich Tel. 0432592479 info[at]medical-board.ch
Wir begrüssen die Stellungnahme der Task Force und insbesondere die entstandene Diskussion. Die Diskussion eröffnet die Möglichkeit, die Position des Swiss Medical Board in Bezug auf den seit über 40 Jahren umstrittenen PSA-Test und dessen Konsequenzen noch einmal differenziert darzustellen und Missverständnisse aus dem Weg zu räumen.

\section{Expertise des Medical Board}

Die Task Force beschreibt das Swiss Medical Board zu Recht als ein interdisziplinäres Gremium. Dieses verfügt mit den Mitgliedern des Expertenrates und der Geschäftsstelle über eine breite Kompetenzbasis. Unerwähnt lässt die Task Force, dass zur Beurteilung der Literatur, der medizinischen Wirkungen und der Kosten des PSA-Tests auch drei externe schweizerische Fachspezialisten (zwei Urologen und ein Labormediziner) beigezogen wurden. Diese renommierten Fachspezialisten wurden auf Vorschlag der FMH (nach Rücksprache mit der Schweizerischen Gesellschaft für Urologie), respektive der FAMH ausgewählt. Wie im Vorwort des Fachberichtes erwähnt, erfolgten die Gesamtwürdigung und die Schlussfassung des Berichts ausschliesslich durch das Medical Board. Insofern müssen die Interpretation der Fakten und die Empfehlungen des Swiss Medical Board nicht notwendigerweise die Meinung der Fachspezialisten wiedergeben. Fakten kann man unterschiedlich interpretieren, sie sollten hingegen möglichst korrekt dargestellt werden.

\section{Unzulänglichkeiten in der PSA-Empfehlung des Swiss Medical Board?}

Die Task Force beschreibt in ihrer Stellungnahme einige Punkte des Fachberichts als «gravierendste Unzulänglichkeiten», ohne darauf hinzuweisen, dass diese vom Swiss Medical Board gleichermassen erkannt und entsprechend dargestellt wurden:

- Eine rein epidemiologische Beurteilung der Studien ohne Bewertung der internen Qualitätskriterien sei ungenügend, insbesondere seien die Quebec-[3], Norrköping-[4] und Stockholm- [5] Studie nicht verwertbar. Das Swiss Medical Board teilt diese Ansicht und hat diese Studien in seinem Bericht auf S. 18 entsprechend als methodisch mangelhaft eingestuft und konsequenterweise nicht für seine Aussagen und Empfehlungen verwertet.

- Die Task Force weist darauf hin, dass die PLCOStudie [6] wegen einer hohen Kontaminationsrate des Kontrollarms statistisch «underpowered» sei. Genau dies wurde im Bericht des Swiss Medical Board dokumentiert (S.19). Zudem wurde auch darauf hingewiesen, dass die ERSCP-Studie [7] ebenfalls nicht frei von methodischen Mängeln ist (S.19).

- Unhaltbar erscheint der Vorwurf der Task Force, dass «die beste Langzeitstudie mit 14 Jahren Follow-up [im Bericht des Medical Board] vernachlässigt wird». Vielmehr ist es so, dass sich die Berechnungen und Aussagen im Bericht des Swiss Medical Board genau auf diese (und nur diese) Studie [8] abstützen (S. 24 des Berichtes). Sowohl das erwähnte Cochrane-Review [9] wie auch die Meta-Analyse von Djulbegovic et al. [10] wurden der Vollständigkeit halber ergänzend dargestellt. Dass die Aussagen und Empfehlungen des Swiss Medical Board auf diesen letztgenannten Arbeiten «basieren», trifft also nicht zu.

- Das Swiss Medical Board teilt die Auffassung der Task Force, dass der in den anderen Studien berichtete Follow-up von 10 Jahren zu kurz ist, um valide Aussagen treffen zu können (S.35 des Berichtes) und ebenso, dass die Angemessenheit 
des PSA-Tests Ende dieses Jahrzehnts auf der bis dahin zu verbessernden Datenlage eventuell neu beurteilt werden muss. Die Ergebnisse einer solchen Neubeurteilung sind derzeit jedoch offen. Auf die angemahnte Berücksichtigung der amerikanischen Mortalitätsstatistiken wurde bewusst verzichtet, weil die sozio-ökonomischen Bedingungen in den USA nicht mit den Schweizer Verhältnissen vergleichbar sind. So ist z. B. die Inzidenz des Prostatakarzinoms in den USA, insbesondere bei der schwarzen Bevölkerung, viel höher als in der Schweiz.

Besonders unverständlich ist, dass die Task Force die labormedizinischen Grenzen der Aussagekraft des PSA-Wertes, die im Bericht des Swiss Medical Board mehrfach und gutbegründet dokumentiert sind (S. 10f, S.20), in keiner Weise erwähnt und kommentiert.

Zusammenfassend sei an dieser Stelle nochmals betont, dass das Swiss Medical Board bei Annahme des günstigsten Szenarios auf Basis der heute zur Verfügung stehenden Evidenz zum Schluss kommt, dass

1. der PSA-Test die krankheitsspezifische Mortalität des Prostatakarzinoms nur geringfügig zu senken vermag und dass

2. angesichts dieser kleinen erwünschten Wirkungen den potentiellen Nachteilen des PSA-Tests, den sog. Downstream-Effekten (Überdiagnostik und Übertherapie) eine erhebliche Bedeutung zukommt.

\section{Die Angemessenheit des PSA-Tests}

Äusserst problematisch ist aus Sicht des Swiss Medical Board die Tatsache, dass sich die Task Force in ihrer Stellungnahme nur auf relative Verhältniszahlen der Mortalität beschränkt und die Bezugsperioden nicht präzisiert. Das Swiss Medical Board folgt den interna-

\section{Besonders unverständlich ist, dass die Task Force die labormedizinischen Grenzen der Aussagekraft des PSA-Wertes in keiner Weise erwähnt und kommentiert.}

tional anerkannten Regeln, wonach zur Beurteilung der Angemessenheit einer Leistung auch absolute Zahlen und klar definierte Bezugsperioden angegeben werden müssen.

Laut Bundesamt für Statistik verstarben im Jahr 2008 in der Schweiz insgesamt 1312 Männer an einem Prostatakarzinom. Dies entspricht einem Anteil von $0,037 \%$ der männlichen Bevölkerung der Schweiz. Laut Angaben der Task Force ereignen sich 14\% dieser Todesfälle bis zum 69. Lebensjahr. Dies entspricht maximal 184 Todesfällen, die potentiell vermeidbar wären (basierend auf der Task Force-Empfehlung, den PSA-Test als Screening-Instrument nur für 50- bis 70-jährige Männer einzusetzen und unter
Vernachlässigung der geringfügigen Effekte auf die Gruppe der Männer über 70 Jahre).

Des Weiteren bezieht sich die Task Force (wie das Swiss Medical Board) ausdrücklich auf «die beste Langzeitstudie mit 14 Jahren Follow-up und 50\% Mortalitätsreduktion». Auch hier empfiehlt es sich, statt der relativen Risikoreduktion die absolute Reduktion des Mortalitätsrisikos aufgrund der Durch-

\section{Äusserst problematisch ist die \\ Tatsache, dass sich die Task Force nur auf relative Verhältniszahlen der Mortalität beschränkt.}

führung des PSA-Tests zu betrachten. Die Originalpublikation [8] berichtet, dass in der Kontrollgruppe 78 Todesfälle infolge eines Prostatakarzinoms beobachtet wurden und in der Screeninggruppe 44 Todesfälle. Betrachtet man hingegen die Gesamtsterblichkeit, so stehen 1982 Todesfällen in der Kontrollgruppe 1981 Todesfälle in der Screeninggruppe gegenüber. In der Gesamtsterblichkeit ist also kein Unterschied zu beobachten! Von den oben genannten 184 Todesfällen könnte der PSA-Test zwar theoretisch 92 Todesfälle pro Jahr verhindern (rechnerisch $184 \times 50 \%=$ 92). Mit Blick auf die Gesamtsterblichkeit wird diese Reduktion aber offenbar vollständig durch andere Todesursachen aufgehoben.

Dass darüber hinaus die mit der Bestimmung des PSA-Wertes einhergehende Überdiagnostik und Übertherapie ein «absolut ernst zu nehmendes und gravierendes Problem» darstellt, hat die Task Force in ihrer Stellungnahme selbst hervorgehoben. Auch die von der Task Force empfohlene «Active Surveillance» bedeutet weitere Konsultationen, Tests, Biopsien und insbesondere eine psychische Belastung

Die Erhebung des PSA-Wertes löst vielfach Folgeuntersuchungen und Therapien aus (Operationen, Bestrahlungen, Medikamente). Nicht selten führen diese zu Nebenwirkungen und Komplikationen wie Inkontinenz, Impotenz und Beeinträchtigung der Darmfunktion. Zusätzlich ist in Erinnerung zu rufen, dass die PSA-Bestimmung unpräzise ist. Auch die gesunde Prostata bildet PSA, und erhöhte Werte können sich aus anderen Gründen ergeben, z. B bei einer Prostataentzündung oder nach der Einnahme von bestimmten Medikamenten. Zudem kann mit dem PSATest nicht unterschieden werden zwischen behandlungsbedürftigen Karzinomen und solchen, die keiner Behandlung bedürfen. Häufig verläuft die Prostatakrebserkrankung bei älteren Patienten sehr langsam und unbemerkt und hat kaum Einfluss auf die Lebensqualität und Lebenserwartung.

Würde der PSA-Test bei allen Männern zwischen 50 und 70 Jahren alle zwei Jahre durchgeführt (bei ei- 
ner angenommenen Partizipationsrate von 70\%), ergäben sich Kosten von 47 Millionen Franken. Berücksichtigt man zusätzlich eventuelle, mit der Durchführung verbundene Folgekosten (z. B. Biopsien) ergäben sich Kosten in der Grössenordnung von 160 Millionen Franken pro Jahr (S.33/34 des Berichts). Derartige Aufwendungen sind immer auch als Opportunitätskosten zu verstehen: Diese Mittel stehen für andere pekte berücksichtigt werden [11, 12, 13]. Versucht man die Empfehlungen der Schweizerischen Gesellschaft für Urologie zu interpretieren, so liegen auch diese - praktisch gesehen - im Bereich einer «Recommendation $\mathrm{C»}$.

Der Bericht des Swiss Medical Board trägt somit dazu bei, das trübe Badewasser zu klären, das Kind bleibt dabei durchaus noch drin!

\section{Die Empfehlungen des Swiss Medical Board entsprechen - ausgedrückt in der Nomenklatur der United States Preventive Services Task Force - insgesamt etwa einer «Recommendation C».}

medizinische Leistungen nicht mehr zur Verfügung, wenn das Prämienvolumen bzw. die Kosten des Gesundheitswesens nicht gleichzeitig erhöht werden sollen. Ähnlich sieht es heute der Entdecker des PSA im Jahre 1970 Richard J. Ablin: «The extraordinary time, effort, and costs associated with the PSA-screening enterprise must be evaluated against other claims on health care spending and physicians' time and energy. We believe that the current PSA-based screening paradigm does not compare favorably with competing health care priorities.» [11].

Wie der Bericht des Swiss Medical Board beleuchtet auch die Stellungnahme der Task Force die Komplexität der vorliegenden Fragestellung. Diese liegt insbesondere darin, dass sich zwei Problemkreise überschneiden - die diagnostische Ungenauigkeit des PSA-Testes und die biologische Heterogenität des Prostatakarzinoms (resp. dessen sehr unterschiedlicher Spontanverlauf). Vor diesem Hintergrund und auf der Basis der bis heute vorliegenden wissenschaftlichen Grundlagen hat das Swiss Medical Board seine Empfehlungen zum PSA-Test bei symptomlosen Männern ohne Risikofaktoren formuliert.

Dabei ist zu beachten, dass diese Empfehlungen in keiner Weise ausschliessen, dass sich der «informierte Mann» mit seinem behandelnden Arzt für die Durchführung eines PSA-Tests entscheiden kann; dann allerdings nicht zulasten der Grundversicherung, wie es im Übrigen der heutigen Rechtslage in der Schweiz entspricht.

Ebenso wird seitens des Swiss Medical Board in keiner Weise bestritten, dass eine besonnene Anwendung des PSA-Testes in der Differentialdiagnose bei urologisch symptomatischen Männern oder in der Therapieverlaufskontrolle ihren Stellenwert hat

Damit entsprechen die Empfehlungen des Swiss Medical Board - ausgedrückt in der Nomenklatur der United States Preventive Services Task Force - insgesamt in etwa einer «Recommendation C». Angesichts der heutigen PSA-Kontroverse ist eine solche Einstufung fair, da neben der momentanen Faktenlage auch die einschlägigen sozial- und individualethischen As-

\section{Literatur}

1 www.medical-board.ch/fileadmin/docs/public/mb/ Fachberichte/2011-10-31_Prostata_Bericht_Final.pdf

2 Recker F, Sulser T. Swisss Medical Board oder: «das Kind mit dem Bade ausschütten». Schweiz Ärztezeitung. 2011;92(51/52):2002-4.

3 Labrie F, Candas B, Cusan L, et al. Screening decreases prostate cancer mortality: 11-year follow-up of the 1988 Quebec prospective randomized controlled trial. Prostate. 2004;59(3):311-8.

4 Sandblom G, Varenhorst E, Rosell J, et al. Randomized prostate cancer screening trial: 20 year followup. BMJ. 2011;1:342.

5 Kjellman A, Akre O, Norming U, et al. 15-year followup of a population based prostate cancer screening study. J Urol. 2009;181(4):1615-21.

6 Andriole GL, Crawford ED, Grubb RL, III, et al. Mortality results from a randomized prostate-cancer screening trial. N Engl J Med. 2009;360(13):1310-9.

7 Schroder FH, Hugosson J, Roobol MJ, et al. Screening and prostate-cancer mortality in a randomized European study. N Engl J Med. 2009;360(13):1320-8.

8 Hugosson J, Carlsson S, Aus G, et al. Mortality results from the Goteborg randomised population-based prostate-cancer screening trial. Lancet Oncol. 2010;11(8):725-32.

9 Ilic D, O'Connor D, Green S, Wilt T. Screening for prostate cancer. Cochrane Database Syst Rev. 2006;3:CD004720.

10 Djulbegovic M, Beyth RJ, Neuberger MM, et al. Screening for prostate cancer: systematic review and meta-analysis of randomised controlled trials. BMJ. 2010;341:c4543.

11 Brett AS, Ablin RJ. Prostate cancer screening - what the U.S. Preventive Services Task Force left out. N Engl J Med. 2011;365(21):1949-51.

12 McNaughton-Collins MF, Barry MJ. One man at a time - resolving the PSA controversy. N Engl J Med. 2011;365(21):1951-3.

13 Schroder FH. Stratiffying risk - the U.S. Preventive Services Task Force and prostate cancer screening. N Engl J Med. 2011;365(21):1953-5. 